ÉGYPTE

monde arabe

\section{Égypte/Monde arabe}

12-13 | 1993

Une économie en transition

\title{
Production d'images négatives et occultation de l'autre
}

\author{
Al-Ahrâm, 11 juillet 1992
}

Nabil ‘Abd al-Fattah

Traducteur : Paul Coatalen

\section{OpenEdition}

1 Journals

Édition électronique

URL : https://journals.openedition.org/ema/1290

DOI : 10.4000/ema.1290

ISSN : 2090-7273

\section{Éditeur}

CEDEJ - Centre d'études et de documentation économiques juridiques et sociales

\section{Édition imprimée}

Date de publication : 31 mars 1993

Pagination : 273-275

ISSN : 1110-5097

\section{Référence électronique}

Nabil 'Abd al-Fattah, «Production d'images négatives et occultation de l'autre », Égypte/Monde arabe [En ligne], 12-13 | 1993, mis en ligne le 08 juillet 2008, consulté le 07 juillet 2022. URL : http:// journals.openedition.org/ema/1290; DOI : https://doi.org/10.4000/ema.1290

Ce document a été généré automatiquement le 7 juillet 2022.

Tous droits réservés 


\title{
Production d'images négatives et occultation de l'autre
}

\author{
Al-Ahrâm, 11 juillet 1992
}

\author{
Nabil 'Abd al-Fattah \\ Traduction : Paul Coatalen
}

1 Assistons-nous, au sein du mouvement culturel et politique égyptien, à la fin de l'histoire des idées comme à celle des méthodes d'analyse et de connaissance ? Ou bien ce qui se passe actuellement dans le domaine de la pensée est-il le symptôme d'une crise intellectuelle? Ce qui nous pousse à nous interroger dans ces termes - en introduction à une étude du phénomène de la violence religieuse reposant sur une discrimination communautaire - c'est le fait que les textes semblent toujours autodestructeurs, et que même ceux qui ont abordé le champ religieux en dehors de la théologie et du clergé se montrent incapables de présenter quelque chose de nouveau, qui tranche par rapport à la littérature dominante.

2 J'incline à répondre à la première question par une affirmative claire, sans ambiguïté, sans l'hésitation qui convient au style universitaire orné de termes abstraits, sans les précautions oratoires qui caractérisent l'objectivité et l'impartialité. Si ce n'est pas à la fin de l'histoire des idées que nous assistons, comment interpréter cette réitération du même langage et des mêmes expressions dans diverses orientations politiques et intellectuelles? La non formation d'un style - en d'autres termes d'une méthode de pensée - et son inertie peuvent-elles signifier autre chose que sa mort? Ces textes unidimensionnels, qui considèrent les effets des phénomènes et les circonstances extérieures des problèmes sans en comprendre les causes, ne reflètent-ils pas une uniformisation à la fois du style de la pensée officielle et de celui de l'opposition? Et si, en fait, elles ne différaient pas l'une de l'autre? Dans l'hypothèse éventuelle de l'accession au pouvoir de l'opposition, le changement politique ne porterait-il pas sur des détails?

3 Comment qualifier l'événement dominant dans la violence et les affrontements interconfessionnels en Égypte? Comment qualifier le rôle rempli par la religion et les interprétations qu'on en fait, ainsi que celui de l'éducation et de la formation dans la 
propagation des conflits idéologiques? Ces phénomènes sont étudiés comme s'ils étaient des évidences, que ce soit la genèse de la fitna (subversion) ou la prévention de celle-ci, sans que personne ne se livre à une analyse ponctuelle de la relation de ces systèmes avec la violence à caractère religieux. Ces phénomènes sont habituellement traités comme des évidences ne méritant pas d'examen. Mais comment savoir s'ils sont vrais ou faux ? La réflexion ne devrait-elle pas prendre en compte une évidence, même si celle-ci est le signe de la démission de la pensée?

4 Dans ces conditions, quel est le non-dit dans tous les monologues actuels traitant de la violence interconfessionnelle égyptienne? Nous nous concentrerons sur deux dimensions parmi d'autres. La première est celle des limites subjectives et cognitives entre les Égyptiens, la deuxième celle de la relation entre le pouvoir de l'exégèse, la crise de la réforme sociale et le renouveau religieux.

\section{Les images ambiguës}

5 La légitimité de la violence dirigée contre un adepte d'une autre religion en Égypte s'explique par plusieurs facteurs structurels parmi lesquels on peut citer l'image de l'autre dans les textes religieux ou encore l'image propagée des deux côtés par les tenants de l'exégèse sacrée avec leurs préjugés, leurs intérêts, leurs passions et leur incitation à un comportement agressif envers l'autre; cela a abouti au contrôle du pouvoir des idéologues du mouvement politico-social par quelques exégètes qui ont également réussi à empêcher toute emprise du mouvement politico-culturel sur l'institution religieuse et fondamentaliste. C'est ce qui permet d'expliquer l'insistance à délimiter subjectivement et objectivement l'identité sociale des croyants ici et celle des autres là-bas, ainsi que la non ouverture des institutions religieuses à la société et aux autres, tout comme l'édification de barrières culturelles et la promotion de valeurs centrées sur le soi qui conduisent à changer l'autre en Satan, ou encore en une entité incompréhensible aux caractéristiques mythico-irrationnelles qui lui retirent son humanité et dont on ne peut attendre que de l'agression ou du moins l'absence de positivité.

6 Ces représentations propagées par les prédicateurs religieux fournissent à rencontre de l'autre des motifs de violence politico-sociale, particulièrement dans les moments de tension. C'est pourquoi les Égyptiens renoncent à l'institution religieuse officielle ou au fondamentalisme extrémiste qui essaie de prendre le pouvoir idéologique et de contrôler les masses égyptiennes des deux côtés. Cette opération trouve dans le champ politico-social un encouragement. Parce que l'événement est fondé sur un essai d'antithèse et la peur de traiter avec le mouvement politico-social sur des bases de classes ou de groupes. La société égyptienne recueille ainsi les fruits de ces dangereuses politiques des fins de siècle.

7 La création de frontières subjectives entre les Égyptiens empoisonne les systèmes d'éducation et d'information qui dévalorisent continuellement l'image de l'autre et l'occultent en insistant sur le fait qu'il faut le traiter dans l'abstrait en niant son humanité et son développement historique. Cela conduit au contrôle suprême qui est entretenu par l'absence de tout dialogue objectif et sérieux dans la société. L'occultation de l'autre est également liée à une valeur négative: celle de l'autoglorification, qui étouffe toute production sociale créatrice pour la communauté. C'est manifestement ce qui est en jeu dans le thème de la défaite et de la décadence 
dans le monde, et dans le désir de se tenir à l'écart de ces maux extérieurs provenant d'entités, de croyances et d'États triomphants qui constituent une présence à la fois permanente et violente et infligent des blessures au moi vaincu. C'est à ce moment-là qu'entrent en jeu l'occultation de l'autre et l'attribution de caractères négatifs à son encontre, ainsi que son rôle de repoussoir pour cimenter la cohésion de l'identité sociale. Cette opération découle de l'affirmation du champ religieux qui est efficace dans l'exacte mesure où il existe un grand vide dans le champ politique et où manquent les fonctions, les outils et les hommes politiques sur la scène sociale.

Il est naturel que ces fonctions se déplacent vers d'autres champs sociaux, comme la mosquée, l'église, la sécurité, la justice et le club, parce que la politique dans la vie moderne est devenue la réplique exacte de la vie sociale et individuelle - elle n'est plus monopolisée entièrement par ceux qui ont le pouvoir - et parce qu'elle fournit des motivations et des besoins qui agissent sur la conscience individuelle et sociale. C'est pourquoi l'individu et le groupe sont entrés sur le marché politique comme producteurs et consommateurs. Lorsque la production politique est absente, ainsi que ses producteurs, le besoin social et individuel de gratification, tout comme les motivations politiques individuelles et collectives, sont transférés dans la sphère religieuse pour satisfaire ces besoins, surtout quand il existe un statut religieux suscitant effectivement des frustrations individuelles et collectives. L'effervescence religieuse s'accroît grâce au pouvoir d'une culture et de valeurs rétrogrades qui entrent en synergie avec l'absence du politique et le grand vide social; tout cela crée envers l'autre une attitude génératrice d'images négatives à son encontre, phénomène qui à son tour alimente le déluge de violence religieuse et factionnelle en Égypte.

INDEX

Mots-clés : violence 